Check for updates

Cite this: RSC Adv., 2019, 9, 29840

\title{
Spacer layer design for efficient fully printable mesoscopic perovskite solar cells $\uparrow$
}

\author{
Tongfa Liu, (D) a Yuli Xiong, ${ }^{\text {b }}$ Anyi Mei, ${ }^{c}$ Yue Hu, (D) ${ }^{c}$ Yaoguang Rong, (D) ${ }^{c d}$ Mi Xu, ${ }^{c}$ \\ Zheng Wang, ${ }^{a}$ Lingyun Lou, ${ }^{a}$ Dongjie Du, ${ }^{a}$ Shizhao Zheng, ${ }^{a}$ Xia Long, ${ }^{a}$ Shuang Xiao, ${ }^{a}$ \\ Shihe Yang (D) *ae and Hongwei Han (D) *c
}

\begin{abstract}
The spacer layer is a key component of fully printable mesoscopic perovskite solar cells, but its precise characteristics are far from being understood in relation to the device design. In the present work, we perform a detailed systematic study on the effects of spacer parameters, such as size of building blocks, layer thickness, etc., on properties of the perovskite filler, insulating ability and performance of fully printable mesoscopic perovskite solar cells by combining the techniques of time-resolved photoluminescence, high-resolution TEM, insulating resistance measurements, impedance spectroscopy and $J-V$ characteristics. Drawing on the deep understanding from these studies, we formulate key principles, which are anticipated to guide the design of the advanced spacer layer for fully printable

mesoscopic perovskite solar cells.
\end{abstract}

Received 13th July 2019

Accepted 23rd August 2019

DOI: $10.1039 / c 9 r a 05357 c$

rsc.li/rsc-advances

Lead halide perovskite (PVSK) as a promising semiconducting material has been introduced as a light harvesting semiconductor because of its ease of fabrication and excellent physical properties, such as tunable bandgap, strong absorbance, long carrier diffusion length and shallow intrinsic trap state level. ${ }^{1-9}$ Extremely flat and compact perovskite thin film with large crystal size has gained particular attention to boost power conversion efficiency (PCE) by sequential deposition method, vapor deposition, solvent-annealing, solvent engineering, hot-casting method, intramolecular exchange methods, and additive, etc. ${ }^{10-16}$ Benefiting from rapid improvements in formation of high quality perovskite thin film, a certified PCE of $25.2 \%$ has been achieved. ${ }^{17}$ However, illumination stability in real environment still remains a serious challenge due to the inherent moisture and UV sensitivity of perovskite. Moreover, using expensive and rare metals as back

\footnotetext{
${ }^{a}$ Guangdong Provincial Key Laboratory of Nano-Micro Materials Research, School of Chemical Biology \& Biotechnology, Peking University Shenzhen Graduate School, Shenzhen, P. R. China. E-mail: chsyang@pku.edu.cn

${ }^{b} H u b e i$ Province Engineering Research Center for Intelligent Micro-nano Medical Equipment and Key Technologies, Wuhan Textile University, Wuhan, Hubei, P. R. China.E-mail: y.xiong@wtu.edu.cn

'Michael Grätzel Center for Mesoscopic Solar Cells, Wuhan National Laboratory for Optoelectronics, China-EU Institute for Clean and Renewable Energy, Huazhong University of Science and Technology, Wuhan, Hubei, P. R. China. E-mail: hongwei. han@mail.hust.edu.cn

${ }^{d}$ School of Physics and Optoelectronics Engineering, Nanjing University of Information Science and Technology, Nanjing, Jiangsu, P. R. China

${ }^{e}$ Department of Chemistry, The Hong Kong University of Science and Technology, Clear Water Bay, Kowloon, Hong Kong 999077, P. R. China

$\dagger$ Electronic supplementary information (ESI) available: Experimental section, Fig. S1-S5 and Table S1. See DOI: 10.1039/c9ra05357c
}

contact, such as gold and silver, may limit large-scale production in the future. As the competing architecture of perovskite solar cells, $\mathrm{TiO}_{2} /$ spacer/carbon (abbreviated as TSC) films based fully printable mesoscopic perovskite solar cells (FP-MPSC) have attracted a lot of researchers due to their low cost and printable large-scale production process. ${ }^{18-21}$ In this type of solar cell, carbon can efficiently collect hole from perovskite layer even without any other hole transporting materials. ${ }^{22,23}$ Most importantly, FP-MPSC could work with excellent illumination stability and heat-stress stability by filling TSC films with (5AVA $)_{x} \mathrm{MA}_{1-x} \mathrm{PbI}_{3}$ (5-AVA = 5-aminovaleric acid, $\mathrm{MA}=$ methylammonium), although the efficiency of $12.8 \%$ is still far behind from the most efficient solar cell. . $^{16,21,24}$

Spacer, as an important part of FP-MPSC, plays a crucial role in obtaining high performance device. Basically, the spacer layer mainly burdens triple important tasks in the efficient mesoscopic perovskite solar cells. Firstly, the core function of spacer is to separate anode and cathode and to prevent electrons in $\mathrm{TiO}_{2}$ from transporting directly to carbon electrode. The separating property of spacer depends on spacer particle sizes, morphology, materials, etc. This requires that spacer layer has no cracking and has wide bandgap. Secondly, the perovskite confined in the mesopores of spacer layer can absorb photons transmitted through perovskite/ $\mathrm{TiO}_{2}$ composite layer and have contribution to photocurrents. Thirdly, the holes produced in the perovskite/ $/ \mathrm{TiO}_{2}$ composite layer have to go through perovskite/spacer composite layer to reach carbon electrode. And the electrons produced in the perovskite/spacer composite layer have to go through perovskite/spacer composite layer to reach $\mathrm{TiO}_{2}$ electrode. Because spacer layer has these important functions, some research on spacer layer have been carried out. 
Recently, $\mathrm{Al}_{2} \mathrm{O}_{3}$ or $\mathrm{ZrO}_{2}$ spacer layer was compared with respect to their pore size. ${ }^{25}$ However, conclusion of the effect of pore size in two different materials was incomplete. The effect of spacer layer thickness was simply discussed both in monolithic dye-sensitized solar cells and FP-MPSC. ${ }^{\mathbf{2 0} 26}$ The morphology of spacer layer was also improved to increase PCE of FP-MPSC. ${ }^{27}$ Although these researches made some progress, there are no clear standards that what should an ideal spacer layer satisfy. Therefore, it is urgent to carry out detailed study on how the parameters of spacer affect the above functions and performance of mesoscopic printable perovskite solar cells.

In the present study, the effects of size of building blocks of spacer layer, thickness of spacer layer on property of perovskite crystals, insulating property, and performance of mesoscopic perovskite solar cells were investigated in details. Based on these deep understandings, critical principles to design advanced spacer layer are proposed.

$\mathrm{ZrO}_{2}$ is used as spacer material due to its large band gap and high conduction band energy level. There are five different sizes of spacer building blocks in this study. The average particle sizes of spacer are measured to be about $5 \mathrm{~nm}, 10 \mathrm{~nm}, 20 \mathrm{~nm}$, $60 \mathrm{~nm}$ and $100 \mathrm{~nm}$, respectively, and hereafter referred to as S5, S10, S20, S60, S100 spacer, respectively. SEM images of asprepared spacer films using these building blocks are shown in Fig. 1. X-ray diffraction patterns of spacer film with different particle sizes are presented in Fig. 1f, indicating that the five spacer films were all tetragonal crystal phase as majority phase. From Scherrer equation, the crystal sizes of spacer building blocks were calculated to be about $5 \mathrm{~nm}, 10 \mathrm{~nm}, 20 \mathrm{~nm}, 30 \mathrm{~nm}$, $30 \mathrm{~nm}$, respectively. These results indicated that the S60 and S100 particles are consisted of $30 \mathrm{~nm}$ sized crystal $\mathrm{ZrO}_{2}$. Fig. 1 presents that there is a large difference in surface morphology with particle size increasing. There are cracks in S5 and S10 spacer films and micrometer scale pores exist in the $\mathrm{S} 100$ spacer, while the surface of S20 and S60 are very uniform without defects.

During solvent evaporation of perovskite precursor, perovskite crystal growth is restricted by randomly interconnected mesopores of spacer film, leading to nanoscale crystal size and
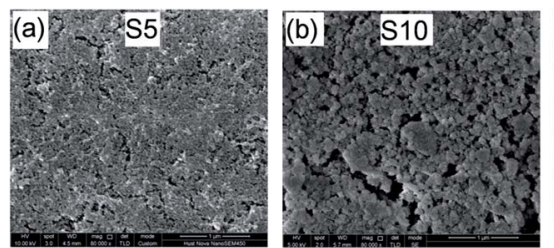

Fig. 2 (a) High resolution transmission electron microscope image of perovskite/S20 spacer film composite. (b) XRD of perovskite/spacer film composite. (c) Steady PL emission spectra and (d) time-resolved $\mathrm{PL}$ of perovskite/spacer film composite.
Fig. 1 SEM images of spacer films with particle size of $5 \mathrm{~nm}$ (a), $10 \mathrm{~nm}$ (b), $20 \mathrm{~nm}$ (c), $60 \mathrm{~nm}$ (d) and $100 \mathrm{~nm}$ (e), respectively. (f) XRD patterns of spacer film with different building block sizes.

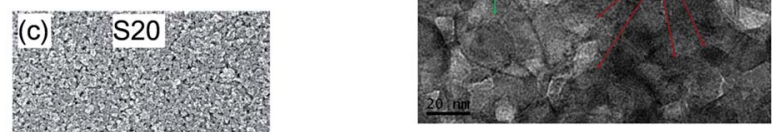

(a)


random crystal orientation, as observed by high resolution ssion electron microscope (Fig. 2a), in which clear spacer particles. Meanwhile, the mesopores of spacer film is fulfilled with perovskite materials, providing continuous channels for charge carriers. The crystal size of perovskite material in spacer film is strongly influenced by mesopore size of spacer layer, as seen in XRD intensity of perovskite at $2 \theta$ of about $14.2^{\circ}$ Fig. 2b). There is an apparent trend that the intensity increased with increasing the particle size of spacer film. As a reference, perovskite was also deposited on bare glass, which exhibited the best crystallinity. The calculated sizes of perovskite from XRD spectra are $4.3 \mathrm{~nm}, 3.9 \mathrm{~nm}, 6.2 \mathrm{~nm}, 11.5 \mathrm{~nm}$, and $12.6 \mathrm{~nm}$, Apparently, the sizes of perovskite crystals confined in the spacer layer are smaller than the average pore sizes of spacer layer measured by $\mathrm{N}_{2}$ absorption/desorption isotherms (Table S1†). The high-resolution TEM image also gives consistent results. For example, the sizes of perovskite crystals confined in the TEM image (Fig. 2a). The infiltrated perovskite started to nucleate onto the heterogeneous surface of spacer building blocks with high surface area, resulting to multiple nucleation crers and small crystal size. In order to evaluate the effect of the band-edge emission spectra of perovskite/spacer composite film were measured in Fig. 2c. The band-edge emission specof perovskite deposited on bare glass peaked at $762 \mathrm{~nm}$ (with photon energy of $1.627 \mathrm{eV}$ ). As the particle size of spacer luminescence occurred, and linewidth broadened. The peak position of perovskite emission spectra can be tuned in the range of $33 \mathrm{~nm}$ through varying the pore size of spacer film. The increase of emission line width at grain boundaries can be attributed to disorder and defects of perovskite, ${ }^{14,15}$ which also led to the decrease of lifetime in time-resolved PL (Fig. 2d).

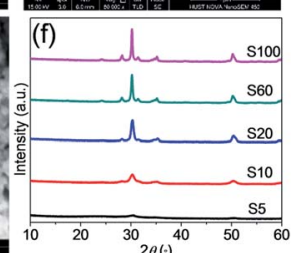


Perovskite film grown on glass has the lifetime of 141.9 ns. However, perovskite grown in spacer film decreased to $0.5 \mathrm{~ns}$, $8.4 \mathrm{~ns}, 24.1 \mathrm{~ns}, 37.2 \mathrm{~ns}, 53.7 \mathrm{~ns}$, for S5, S10, S20, S60, S100, respectively.

The particle size of spacer also has large effect on insulating ability of spacer films with the same thickness. FTO/spacer/ carbon configuration was designed to measure the insulating ability of spacer layers. In ideal conditions, the resistance between carbon and FTO, defined as insulating resistance $\left(R_{\mathrm{I}}\right)$, should be infinite, indicating that there is not any leakage current from ideal insulating spacer. However, all of the measured resistance has finite values, summarized in Table 1, from $300 \Omega$ to $1.5 \mathrm{k} \Omega$. There are clear trends of insulating resistance with particle sizes of spacer. The S100 spacer film has lowest insulating resistance, indicating the poorest insulating ability. It was found that the S100 spacer film was very loosely packed even after sintering at 500 degrees for one hour. The sintered S100 spacer film could be easily peeled off from substrate using 3M Scotch tape. In contrast, S5 and S10 spacer films were much more robust than other spacer films to be peeled off from the substrate after sintering at 400 degrees for one hour. This difference in spacer film about peeling strength was resulted from sintering energetics and was originated from quantum size effect dominated by surface to bulk atom ratio of spacer building block nanocrystals. ${ }^{28-31}$

Fig. $3 \mathrm{a}$ is the scheme showing the layout of FP-MPSC. The influence of the size of spacer layer building block on the photocurrent density-voltage $(J-V)$ curves of the (5-AVA $)_{x}$ $\mathrm{MA}_{1-x} \mathrm{PbI}_{3}$ perovskite devices without and with spacer layer was evaluated in Fig. $3 \mathrm{~b}$ and photovoltaic parameters are summarized in Table 1. FP-MPSC without spacer was studied as the reference device. The device without spacer film has a relatively low efficiency of $6.52 \%$, featuring very low open-circuit voltage $\left(V_{\mathrm{OC}}\right)$ of $605 \mathrm{mV}$, which is consistent with previous result for the devices using two-step method deposited $\mathrm{MAPbI}_{3}$ perovskite. $^{\mathbf{2 0}}$ The $V_{\text {OC }}$ increased significantly from $605 \mathrm{mV}$ to above $808 \mathrm{mV}$, when the device added spacer layers. The device with S100 spacer, which had best crystallinity and longest photoluminescence lifetime, is expected to have excellent performance. But S100 spacer has relatively low $V_{\text {OC }}$ of $808 \mathrm{mV}$ and low PCE of $10.10 \%$. This is resulted from poorest insulating ability of S100 spacer among these spacer films. Balancing from photoluminescence property of perovskite confined in spacer layer and insulating property of spacer layer, $20 \mathrm{~nm}$-sized S20 spacer film had the best performance with efficiency of $11.86 \%$. It is
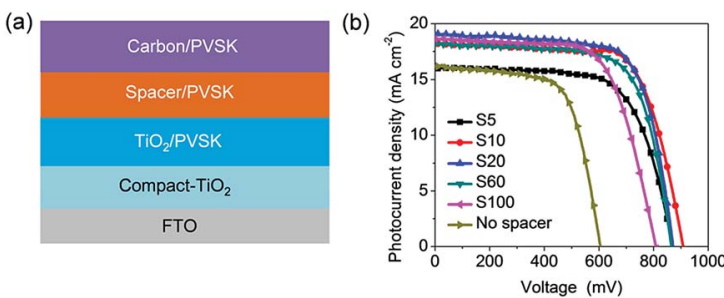

Fig. 3 (a) Scheme showing the layout of FP-MPSC. (b) J-V curves of devices based on spacer film with different building block sizes.

common sense that devices with large perovskite crystal size with low trap density can approach $V_{\text {OC }}$ to the limit of theoretical value. ${ }^{1,32,33}$ The blue shift of the band-edge photoluminescence and decreased lifetime of perovskite in spacer film may explain the relatively low $V_{\mathrm{OC}}$ (less than $1.0 \mathrm{~V}$ ) relative to conventional planar perovskite solar cells ( $V_{\text {OC }}$ was more than $1.1 \mathrm{~V}$ ) in which the size of perovskite crystal was larger than $500 \mathrm{~nm}$. Spacer film with large pore size and excellent insulating property are expected to further improve $V_{\mathrm{OC}}$ of FP-MPSC. The observed quantum size effect of perovskite nanocrystals confined in spacer mesopores in some extents results from templating effect of spacer nanoparticles and unmatched crystal lattices. Matched crystal lattices may mitigate the quantum size effect by heteroepitaxy via reduced nucleation density. ${ }^{34}$

The effect of thickness of spacer film on mesoscopic perovskite solar cells was briefly discussed in our previous study. ${ }^{20,35}$ Here, detailed study from the points of insulating ability and impedance was carried out to clarify the mechanism on how spacer film affects the performance of FP-MPSC.

All photovoltaic parameters depended on the thickness of spacer film (Fig. 4a). Dark current (Fig. 4b) is suppressed by increasing thickness of spacer film, which is in good agreement with that $V_{\mathrm{OC}}$ reached maximum value and remained stable when thickness is above $2.64 \mu \mathrm{m}$. The $V_{\text {OC }}$ has coincident trends with insulating resistance in Fig. 4c. Therefore, $V_{\text {OC }}$ has strong relationship with insulating ability of spacer film when other conditions are the same. $J_{\mathrm{SC}}$ reached maximum value and remained stable when thickness is above $3.31 \mu \mathrm{m}$ by harvesting more photons. $J_{\mathrm{SC}}$ decreased with over $5 \mu \mathrm{m}$-thick spacer layer resulted from recombination. PCE reached maximum value when thickness is about $4.84 \mu \mathrm{m}$. The device without spacer film has poorest $V_{\text {OC }}$, so there is a need to avoid the damage of spacer film when screen printing of carbon film onto spacer

Table 1 Photovoltaic parameters of mesoscopic perovskite solar cells based on spacer with different building block sizes

\begin{tabular}{|c|c|c|c|c|c|c|}
\hline Spacer & Lifetime (ns) & $R_{\mathrm{I}}(\Omega)$ & $J_{\mathrm{SC}}\left(\mathrm{mA} \mathrm{cm}^{-2}\right)$ & $V_{\mathrm{OC}}(\mathrm{mV})$ & $\mathrm{FF}(\%)$ & PCE (\%) \\
\hline No spacer & - & 30 & 16.21 & 605 & 66 & 6.52 \\
\hline S5 & 0.5 & 1500 & 16.06 & 871 & 67 & 9.42 \\
\hline S20 & 24.1 & 960 & 19.10 & 871 & 71 & 11.86 \\
\hline S60 & 37.2 & 800 & 18.19 & 865 & 70 & 11.08 \\
\hline $\mathrm{S} 100$ & 53.7 & 300 & 18.62 & 808 & 67 & 10.10 \\
\hline
\end{tabular}



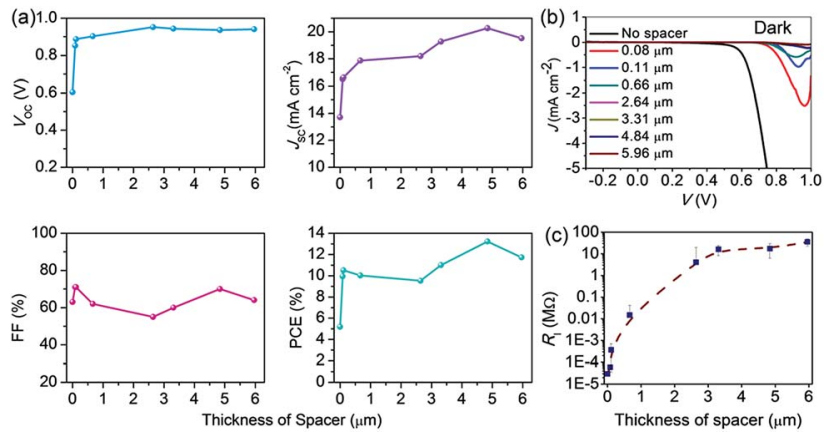

$V(\mathrm{~V})$

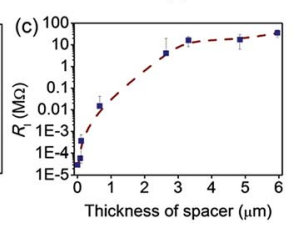

Fig. 4 (a) Photovoltaic parameters dependence on thickness of S20 spacer film. (b) Dark current of FP-MPSC based on different thickness of spacer film. (c) Insulating resistance $\left(R_{1}\right)$ of spacer film with different thickness.

film. The dependence of $J_{\mathrm{SC}}$ on thickness of spacer film and different trends between $J_{\mathrm{SC}}$ and $V_{\mathrm{OC}}$ indicate that perovskite confined in spacer film can generate charge carriers, playing similar role of perovskite capping layer in conventional mesoporous/planar bilayer perovskite solar cells. The fill factor (FF) was not linearly decreased when thickness of spacer film was increased, although the transporting distance of charge carriers was increased. This result was different from dyesensitized solar cells, where FF was linearly decreased when thickness of spacer film was increased. ${ }^{26}$

To further elucidate the relation between thickness of the spacer film and the photovoltaic performance, impedance spectra (IS) were measured. Before analyzing the spectra, physical process correlated to each semicircle should be identified qualitatively. Up to now, IS analysis on fully printable mesoscopic perovskite solar cells simply applied existing models of dye-sensitized solar cells or planar perovskite solar cells. To assure reliability, IS are analyzed by varying thickness of spacer film, with or without meso- $\mathrm{TiO}_{2}$ to assign high frequency and low frequency semicircles. The photographs of devices used to measure the IS are shown in Fig. S1 and S2. $\dagger$ The typical Nyquist plot and Bode plot of the FP-MPSC device with different spacer film thickness measured at $0.3 \mathrm{~V}$, under weak illumination was plotted in Fig. 5 . The full IS can be found in Fig. S3 and S4, in the ESI. $\dagger$ The IS in high frequency part was modelled with one resistance paralleling with one constant phase element and adding another series resistance, as depicted in Fig. S5. $\dagger$ The fitting resistance and capacitance was
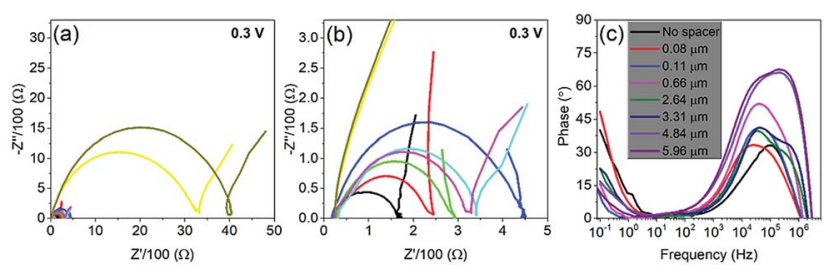

Fig. 5 Nyquist plot ( $a$ and $b$ ) and Bode plot (c) of the FP-MPSC device with different spacer film thickness measured at $0.3 \mathrm{~V}$, under weak illumination (0.1 sun). normalized with active area, as presented in Fig. 6. Under weak light ( 0.1 sun), series resistances $\left(R_{\mathrm{s}}\right)$ are almost between 10-15 $\Omega \mathrm{cm}^{2}$, remaining constant in the whole bias voltage range while high frequency resistances $\left(R_{\text {Perovskite }}\right)$ increased clearly with increasing thickness of spacer film and associating capacitance decreased with increasing thickness of spacer film. The change in high frequency semicircle is not related to carbon/perovskite interface because the contact of carbon/ perovskite interface was unchanged. Therefore, the change in high frequency semicircle is originated from thickness varying of spacer film. Integrating the above features, it is concluded that low frequency semicircle is related to $\mathrm{TiO}_{2} /$ perovskite interface, which is in good agreement with the usual understanding that charge carrier recombination in $\mathrm{TiO}_{2} /$ perovskite interface is slow process, and high frequency semicircle is related to both perovskite confined in spacer film and carbon/ perovskite interface. This was consistent with other researcher's results in which one extra feature related to perovskite was observed from high to intermediate frequency. ${ }^{36}$ The new feature leads to abrupt decrease of phase value of constant phase element below $0.4 \mathrm{~V}$, as can be clearly seen in Fig. 6d. The new feature is also clear in the Nyquist plot and Bode plot in Fig. 5b and c, where two RC circuit components merge at high frequency $\left(10^{4}-10^{5} \mathrm{~Hz}\right)$. One simple model to interpreting this new feature is to consider the perovskite/ spacer composite layer as a standard parallel plate capacitor, and the capacitance can be written as eqn (1).

$$
C=\varepsilon_{0} \varepsilon S / d
$$

where $\varepsilon_{0}$ is vacuum dielectric constant, $\varepsilon$ is relative dielectric constant of perovskite, $S$ is the active area of device, and $d$ is the thickness of spacer layer. Because the spacer layer is wide bandgap materials, there is little charge in the spacer itself. The charge transport is mainly from perovskite confined in the
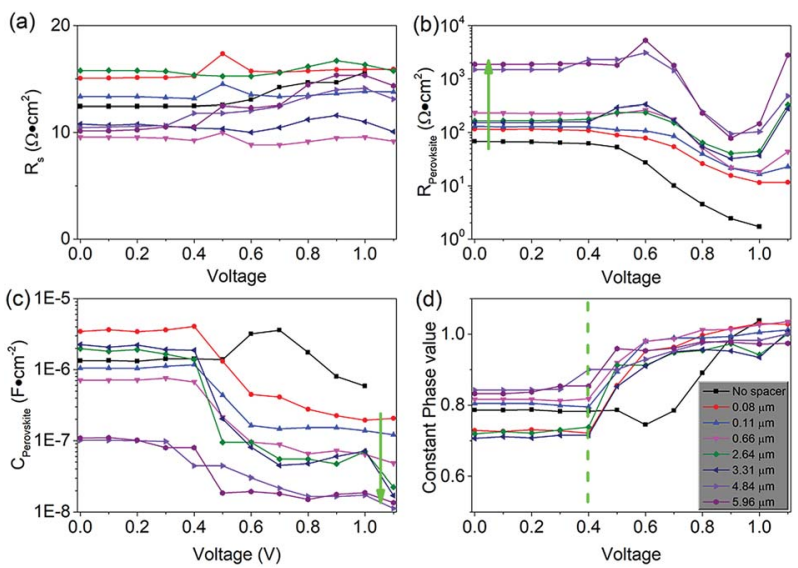

Fig. 6 Parameters obtained from high frequency $\left(\sim 10^{2}-10^{6} \mathrm{~Hz}\right)$ semicircle IS analysis of the FP-MPSC device with different spacer film thickness measured at between $1.1 \mathrm{~V}$ and $0 \mathrm{~V}$, under weak illumination (0.1 sun). (a) Series resistance. (b) Resistance related to perovskite. (c) Capacitance related to perovskite and (d) associated constant phase value. 
spacer layer. The normalized capacitance with active area will be reciprocal of $d$, which is in good consistent with the trend of fitting capacitance ( $R_{\text {Perovskite }}$ in Fig. 6c). Based on the above results, it can be concluded that the extra feature in high to intermediate frequency is resulting from charge transport of perovskite confined in the spacer layer. This conclusion is very consistent with the analysis of the geometrical capacitance of the perovskite layer in the planar perovskite solar cells. ${ }^{37}$ The thick spacer layer of over $2.5 \mu \mathrm{m}$ with enough insulating ability would definitely increase the charge carrier transport length through the spacer layer and cause severe recombination considering the relatively smaller perovskite nanocrystals.

Combining the analysis about the effect of building block size and thickness of spacer layer, the bottlenecks of current spacer layer and infiltrated perovskite are summarized in left picture of Fig. 7. Perovskite layer embedded in mesoporous matrix of spacer layer are usually composed of crystals with size of tens of nanometers. Although observed quantum size effect may have useful application in LED or other optoelectronic fields, the limited charge transport ability in quantum dots is detrimental for achieving high PCE in photovoltaic device due to serious charge carrier recombination. Moreover, the thickness of spacer layer has to be several micrometers to insure enough insulating ability, which further hinders the charge transport to charge carrier selective layer. These two unfavorable factors decrease the potentially achievable PCE. It has been proved that grain boundaries are not beneficial for attaining high performance perovskite solar cells. ${ }^{1}$ Monolayer perovskite crystals in vertical direction are usually deposited on chargeselective layer in order to reduce recombination near grain boundary. According to the absorption coefficient of $\mathrm{MAPbI}_{3}$ perovskite, $1 \mu \mathrm{m}$ thick $\mathrm{MAPbI}_{3}$ perovskite layer can absorb over $95 \%$ incident light at wavelength of $750 \mathrm{~nm} .{ }^{15,33}$ Fixing the porosity of spacer layer to be $40 \%, 2.5 \mu \mathrm{m}$ thick spacer layer has equivalent $1 \mu \mathrm{m}$ thickness of perovskite layer. Considering that the size of perovskite crystals is less than $100 \mathrm{~nm}$, the ratio of length of spacer thickness to size of perovskite crystals is over 25 , which means that charge generated in spacer/perovskite layer has encountered at least 25 grain boundaries before reaching charge selective layer. To overcome these issues, the ideal features of spacer layer and infiltrated perovskite are illustrated in right picture of Fig. 7. Porous single-crystal perovskite models grown in matrix of porous spacer template

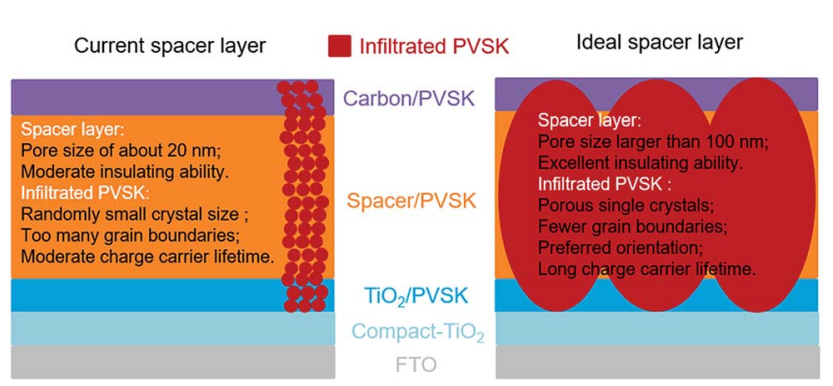

Fig. 7 Illustration to show problems of current spacer layer and infiltrated perovskite, and proposed features of ideal spacer layer and infiltrated perovskite. with 2D arrays or 3D of structured films are more favorable for achieving high PCE. ${ }^{38}$ Perovskite nanorod has high mobility due to confined charge transport. It is known in the zeolite field that meso-crystal materials can grow from one ordered template, which needs controlled template-precursor interaction. ${ }^{39-41}$ In fact, porous single crystal $\mathrm{MAPbI}_{3}$ has been realized via additive in perovskite precursor solution. ${ }^{42}$ Some favorable phenomena has been also observed in investigating the crystallization of perovskite in mesopores of TSC films. For example, the crystal growth with preferential orientation was realized via moistureinduced crystallization process in the $\mathrm{NH}_{4} \mathrm{Cl}$-PVSK complex. ${ }^{43}$ Even the perovskite crystal growth was significantly templated by scaffold, pronounced positive effect was occurred in photovoltaic performance. Therefore, exploring methods to grow mesoporous single-crystal perovskite with less grain boundaries and higher mobility in ordered spacer layer would further promote PCE of FP-MPSC device.

Another issue to overcome is the unideal insulating ability of spacer film. The melting point of $\mathrm{ZrO}_{2}$ is about 2700 degrees. Sub-micrometer to nanometer $\mathrm{ZrO}_{2}$ is usually used to decrease the sintering temperature below 1000 degrees in the ceramic field. ${ }^{31}$ The particle size of $\mathrm{ZrO}_{2}$ has to be small enough to be sintered at allowed temperatures (the FTO glass will soften when $T>550$ degrees). ${ }^{44}$ The insulating ability is mainly associated with porosity and interparticle connection. The porosity is similar for all particle size. The different insulating ability arises mainly from interparticle connection. To improve insulating ability, low-melting point wide bandgap nanomaterials is preferred as building blocks. Aluminum oxide, silica, or ternary oxides may provide better choice as spacer building blocks. ${ }^{\mathbf{4 5}}$ Recently, by depositing a thin layer of $\mathrm{Al}_{2} \mathrm{O}_{3}$ onto surface of mesoporous $\mathrm{TiO}_{2}$ before printing spacer layer, the $\mathrm{ZrO}_{2}$ thickness was reduced from $3 \mu \mathrm{m}$ to $1.2 \mu \mathrm{m}$ while retaining comparable device performance. ${ }^{35}$

\section{Conclusions}

In this work, we have studied the effects of size of spacer building blocks, spacer thickness on optoelectronic property of perovskite, insulating property, and performance of FP-MPSC in details. The time-resolved PL and the band-edge emission spectrum of perovskite depends greatly on particle size of spacer building blocks. It was also found that the open-circuit voltage has clear relationship with insulating ability of spacer layer through varying spacer layer thickness in a large range. The charge transport feature in spacer layer is discussed with impedance spectra. Based on these deep understandings, it was proposed that advanced spacer film features pore size larger than $100 \mathrm{~nm}$, high porosity and simultaneously ideal insulating ability with film thickness as thin as possible. Reasonable routes to realize ideal spacer layer are also proposed. The results will promote spacer improvement of FP-MPSC in the near future.

\section{Conflicts of interest}

There are no conflicts to declare. 


\section{Acknowledgements}

The authors acknowledge the financial support of this work from the National Natural Science Foundation of China (Grant No. 51502141, 91733301, 91433203, 61474049, 51773230, and 21702069), the Ministry of Science and Technology of China (863, 2015AA034601), the Fundamental Research Funds for the Central Universities (No. 2019kfyXJJS051), the Program for HUST Academic Frontier Youth Team, the Science and Technology Department of Hubei Province (No. 2017AAA190), the 111 Project (No. B07038), the Double first-class research funding of China-EU Institute for Clean and Renewable Energy (No. ICARE-RP-2018-SOLAR-001 and ICARE-RP-2018-SOLAR-002), the Shenzhen Peacock Plan (KQTD2016053015544057), the Nanshan Pilot Plan (LHTD20170001), the NFSC/Hong Kong RGC Research Scheme (N_HKUST610/14), the Guangdong Science and Technology Program (2017B030314002), the HKRGC General Research Funds (GRF No. 16312216), the HK Innovation and Technology Fund (ITS/219/16 and GHP/079/ 17SZ), and China Postdoctoral Science Foundation Grant (No. 2017M612452 and 2018M631240). We thank the Analytical and Testing Centre of Huazhong University of Science and Technology for field emission SEM testing.

\section{Notes and references}

1 C. Bi, Q. Wang, Y. Shao, Y. Yuan, Z. Xiao and J. Huang, Nat. Commun., 2015, 6, 7747.

2 Q. Dong, Y. Fang, Y. Shao, P. Mulligan, J. Qiu, L. Cao and J. Huang, Science, 2015, 347, 967-970.

3 F. Hao, C. C. Stoumpos, R. P. H. Chang and M. G. Kanatzidis, J. Am. Chem. Soc., 2014, 136, 8094-8099.

4 N. Kitazawa, Y. Watanabe and Y. Nakamura, J. Mater. Sci., 2002, 37, 3585-3587.

5 A. Kojima, K. Teshima, Y. Shirai and T. Miyasaka, J. Am. Chem. Soc., 2009, 131, 6050-6051.

6 H. Shi and M.-H. Du, Phys. Rev. B: Condens. Matter Mater. Phys., 2014, 90, 174103.

7 W. J. Yin, T. Shi and Y. Yan, Adv. Mater., 2014, 26, 4653-4658.

8 W.-J. Yin, T. Shi and Y. Yan, Appl. Phys. Lett., 2014, 104, 063903.

9 H. S. Kim, C. R. Lee, J. H. Im, K. B. Lee, T. Moehl, A. Marchioro, S. J. Moon, R. Humphry-Baker, J. H. Yum, J. E. Moser, M. Gratzel and N. G. Park, Sci. Rep., 2012, 2, 591.

10 J. Burschka, N. Pellet, S.-J. Moon, R. Humphry-Baker, P. Gao, M. K. Nazeeruddin and M. Grätzel, Nature, 2013, 499, 316319.

11 N. J. Jeon, J. H. Noh, Y. C. Kim, W. S. Yang, S. Ryu and S. I. Seok, Nat. Mater., 2014, 13, 897-903.

12 X. Li, M. I. Dar, C. Y. Yi, J. S. Luo, M. Tschumi, S. M. Zakeeruddin, M. K. Nazeeruddin, H. W. Han and M. Gratzel, Nat. Chem., 2015, 7, 703-711.

13 M. Liu, M. B. Johnston and H. J. Snaith, Nature, 2013, 501, 395-398.

14 W. Nie, H. Tsai, R. Asadpour, J. C. Blancon, A. J. Neukirch, G. Gupta, J. J. Crochet, M. Chhowalla, S. Tretiak,
M. A. Alam, H. L. Wang and A. D. Mohite, Science, 2015, 347, 522-525.

15 Z. Xiao, Q. Dong, C. Bi, Y. Shao, Y. Yuan and J. Huang, Adv. Mater., 2014, 26, 6503-6509.

16 W. S. Yang, J. H. Noh, N. J. Jeon, Y. C. Kim, S. Ryu, J. Seo and S. I. Seok, Science, 2015, 348, 1234-1237.

17 Best Research-Cell Efficiencies, https:/www.nrel.gov/pv/ assets/pdfs/best-research-cell-efficiencies.20190802.pdf, 2019.

18 Z. Ku, Y. Rong, M. Xu, T. Liu and H. Han, Sci. Rep., 2013, 3, 03132.

19 L. F. Liu, A. Y. Mei, T. F. Liu, P. Jiang, Y. S. Sheng, L. J. Zhang and H. W. Han, J. Am. Chem. Soc., 2015, 137, 1790-1793.

20 T. Liu, L. Liu, M. Hu, Y. Yang, L. Zhang, A. Mei and H. Han, J. Power Sources, 2015, 293, 533-538.

21 A. Mei, X. Li, L. Liu, Z. Ku, T. Liu, Y. Rong, M. Xu, M. Hu, J. Chen, Y. Yang, M. Gratzel and H. Han, Science, 2014, 345, 295-298.

22 Z. Wei, K. Yan, H. Chen, Y. Yi, T. Zhang, X. Long, J. Li, L. Zhang, J. Wang and S. Yang, Energy Environ. Sci., 2014, 7, 3326-3333.

23 K. Yan, Z. Wei, J. Li, H. Chen, Y. Yi, X. Zheng, X. Long, Z. Wang, J. Wang, J. Xu and S. Yang, Small, 2015, 11, 22692274.

24 X. Li, M. Tschumi, H. W. Han, S. S. Babkair, R. A. Alzubaydi, A. A. Ansari, S. S. Habib, M. K. Nazeeruddin, S. M. Zakeeruddin and M. Gratzel, Energy Technol., 2015, 3, 551-555.

25 Z. Meng, D. P. Guo, J. G. Yu and K. Fan, Appl. Surf. Sci., 2018, 430, 632-638.

26 H. Wang, G. Liu, X. Li, P. Xiang, Z. Ku, Y. Rong, M. Xu, L. Liu, M. Hu, Y. Yang and H. Han, Energy Environ. Sci., 2011, 4, 2025.

27 T. Liu, Y. Rong, Y. Xiong, A. Mei, Y. Hu, Y. Sheng, P. Jiang, X. Hou, M. Duan, Y. Guan, L. Hong and H. Han, RSC Adv., 2017, 7, 10118-10123.

28 E. A. Barringer and H. K. Bowen, J. Am. Ceram. Soc., 1982, 65, C-199-C-201.

29 C. T. Campbell, S. C. Parker and D. E. Starr, Science, 2002, 298, 811-814.

30 W. H. Rhodes, J. Am. Ceram. Soc., 1981, 64, 19-22.

31 I.-W. Chen and X.-H. Wang, Nature, 2000, 404, 168.

32 Y. Shao, Y. Yuan and J. Huang, Nat. Energy, 2016, 1, 1-6.

33 H. Chen, F. Ye, W. T. Tang, J. J. He, M. S. Yin, Y. B. Wang, F. X. Xie, E. B. Bi, X. D. Yang, M. Gratzel and L. Y. Han, Nature, 2017, 550, 92-95.

34 L. Ji, H. Y. Hsu, J. C. Lee, A. J. Bard and E. T. Yu, Nano Lett., 2018, 18, 994-1000.

35 Q. Wang, S. Liu, Y. Ming, Y. Guan, D. Li, C. Zhang, Z. Wang, Y. Rong, Y. Hu and H. Han, Sustainable Energy \& Fuels, 2018, 2, 2412-2418.

36 A. Dualeh, T. Moehl, N. Tetreault, J. Teuscher, P. Gao, M. K. Nazeeruddin and M. Gratzel, ACS Nano, 2014, 8, 4053. 37 A. Guerrero, G. Garcia-Belmonte, I. Mora-Sero, J. Bisquert, Y. S. Kang, T. J. Jacobsson, J.-P. Correa-Baena and A. Hagfeldt, J. Phys. Chem. C, 2016, 120, 8023-8032. 
38 E. J. Crossland, N. Noel, V. Sivaram, T. Leijtens, J. A. Alexander-Webber and H. J. Snaith, Nature, 2013, 495, 215-219.

39 C. Dickinson, W. Zhou, R. P. Hodgkins, Y. Shi, D. Zhao and H. He, Chem. Mater., 2006, 18, 3088-3095.

40 K. Jiao, B. Zhang, B. Yue, Y. Ren, S. Liu, S. Yan, C. Dickinson, W. Zhou and H. He, Chem. Commun., 2005, 5618-5620.

41 C. J. H. Jacobsen, C. Madsen, J. Houzvicka, I. Schmidt and A. Carlsson, J. Am. Chem. Soc., 2000, 122, 7116-7117.
42 T. Kollek, D. Gruber, J. Gehring, E. Zimmermann, L. Schmidt-Mende and S. Polarz, Angew. Chem., Int. Ed., 2015, 54, 1341-1346.

43 Y. Rong, X. Hou, Y. Hu, A. Mei, L. Liu, P. Wang and H. Han, Nat. Commun., 2017, 8, 14555.

44 J.-C. Lee, T. G. Kim, W. Lee, S.-H. Han and Y.-M. Sung, Cryst. Growth Des., 2009, 9, 4519-4523.

45 Y. Xiong, X. Zhu, A. Mei, F. Qin, S. Liu, S. Zhang, Y. Jiang, Y. Zhou and H. Han, Sol. RRL, 2018, 2, 1800002. 